\title{
Exploring Dyson's Orbitals and Their Electron Binding Energies for Conceptualizing Excited States from Response Methodology
}

\author{
Vladimir Pomogaev, Seunghoon Lee, Sason Shaik,* Michael Filatov,* and Cheol Ho Choi*
}

Cite This: J. Phys. Chem. Lett. 2021, 12, 9963-9972

Read Online

ABSTRACT: The molecular orbital (MO) concept is a useful tool, which relates the molecular ground-state energy with the energies (and occupations) of the individual orbitals. However, analysis of the excited states from linear response computations is performed in terms of the initial state MOs or some other forms of orbitals, e.g., natural or natural transition orbitals. Because these orbitals lack the respective energies, they do not allow developing a consistent orbital picture of the excited states. Herein, we argue that Dyson's orbitals enable description of the response states compatible with the concepts of molecular orbital theory. The Dyson orbitals and their energies obtained by mixed-reference spin-flip time-dependent density functional theory (MRSF-TDDFT) for the response ground state are remarkably similar to the canonical MOs obtained by the usual DFT calculation. For excited states, the Dyson orbitals provide a chemically sensible picture of the electronic transitions, thus bridging the chasm between orbital theory and response computations.

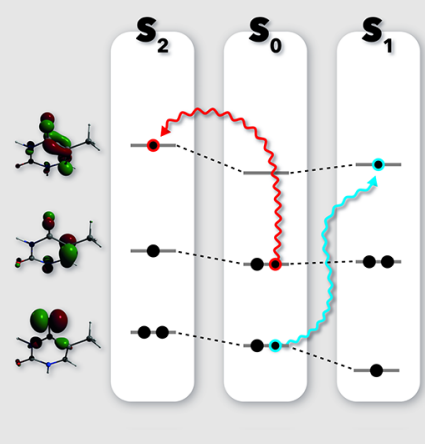

$T^{1}$ he concept of molecular orbitals ${ }^{1-4}$ is a cornerstone of modern chemical theory. ${ }^{5}$ Ranging from simple qualitative theories ${ }^{2,6}$ to full self-consistent field (SCF) methodologies, $^{4,7-10}$ the molecular orbitals (MOs) provide a lucid representation of the electronic structure of molecules. ${ }^{11} \mathrm{~A}$ considerable advantage of the SCF MO theory is that the eigenvalues of the molecular Lagrangian matrix (the MO energies) approximate the ionization energies of the molecule, ${ }^{12}$ thus providing a bridge between abstract theory and physical observation.

In its SCF (or mean-field) implementation, the MO theory misses the electron correlation effects, ${ }^{13}$ which are introduced either through the use of configurational expansions (e.g., the method of configuration interaction $)^{14}$ or through the use of density functional theory (DFT) ${ }^{15,16}$ Although the KohnSham (KS) ${ }^{16}$ DFT retains the simplicity of a mean-field theory, the KS orbital energies should provide an improved approximation to the exact ionization potentials, ${ }^{17,18}$ at least at the formal level.

At the level of correlated theory, analysis of the many-body wave functions is often carried out in terms of the natural orbitals (NOs), which are defined as the eigenfunctions of the true one-particle density matrix of the many-body system. ${ }^{19,20}$ Although the NOs are supposed to provide the most compact expansion of many-body wave functions, ${ }^{21-23}$ they cannot be straightforwardly connected with observable properties, such as the ionization energies. As there are no energy levels associated with NOs, the usual chemical concepts (e.g., the Aufbau principle, ${ }^{24,25}$ the Woodward-Hoffmann rules, ${ }^{26,27}$ frontier orbital theory, ${ }^{28}$ etc.) or the band structure of crystalline solids cannot be straightforwardly formulated in terms of NOs.

The excited electronic states of molecules are often analyzed in terms of the orbital transitions, e.g., the highest occupied $\mathrm{MO}(\mathrm{HOMO}) \rightarrow$ the lowest unoccupied MO (LUMO) transition. Such an analysis is feasible only in the case when one orbital transition dominates the excited state. ${ }^{29}$ However, quite often, especially when the orbital relaxation effects are strong, the excited state is represented by a superposition of multiple orbital transitions, and its analysis in terms of the orbitals of the initial, e.g., the ground, electronic state becomes unwieldy. Although the use of the natural transition orbitals ${ }^{30}$ (NTOs) obtained by singular value decomposition of the transition density matrix may simplify the analysis of the excited states, ${ }^{29}$ their connection with the orbitals of the initial state is not straightforward. ${ }^{31}$

Generally, the orbitals, be it MOs, NOs, or NTOs, are not experimentally observable. ${ }^{32-36}$ However, it is believed that the information on Dyson's orbitals (DOs) can be accessed experimentally, e.g., inferred from the electron momentum $(\mathrm{e}, 2 \mathrm{e})$ spectroscopy. ${ }^{37,38}$ Indeed, the knowledge of the phase and the density distribution may help to sketch the orbital. ${ }^{36}$ However, this does not mean that this will be the same oneelectron function that governs motion of the electron prior to

Received: July 30, 2021

Accepted: September 22, 2021

Published: October 7, 2021 
its ejection from an atom or a molecule. ${ }^{36}$ Electron removal is a time-dependent process, during which the molecular (or atomic) wave function undergoes a rapid (on an attosecond time scale) transformation between the initial (e.g., neutral) and the final (e.g., cation and a free electron) state. Hence, to extract from the experimental data the information on the orbitals existing prior to the measurement would require the knowledge of the time-evolution. At the theoretical level, the DOs are evaluated within the sudden approximation, ${ }^{39,40}$ which ignores the time-evolution and replaces the ionization amplitude by the overlap integral between the final and the initial states. Hence, the theoretical DOs and the orbitals deducible from the experimental measurements are not exactly the same objects. ${ }^{36}$ Nevertheless, the DOs have a number of features which make them useful for analyzing the molecular electronic structure. ${ }^{41,42}$

Dyson's orbital (or Feynman-Dyson amplitude) $\varphi_{k}$ is defined as the overlap between the many-electron wave functions of the initial state $\Psi^{N}$ (e.g., neutral molecule) and the final state (e.g., ionized molecule) $\Psi_{k}^{N-1}$

$$
\varphi_{k}=\sqrt{N}\left\langle\Psi_{k}^{N-1} \mid \Psi^{N}\right\rangle
$$

where $N$ is the number of electrons in the initial state; $k$ labels the excitation level of the final state, and the integration runs over the coordinates of $N-1$ electrons. ${ }^{36,41-46}$ Dyson's orbitals are not normalized on the usual space metric, and their norms

$$
\left|\gamma_{k}\right|^{2}=\left\langle\varphi_{k} \mid \varphi_{k}\right\rangle
$$

are related with the probability of the given ( $k$ th) ionization channel. ${ }^{41,42,46-49}$ Hence, $\left|\gamma_{k}\right|^{2}$ are often called probability factors or pole strengths; see ref 42 and references cited therein.

A nice feature of Dyson's orbitals is that they are fully compatible with the main concepts of quantum chemistry and offer opportunity to analyze the properties of many-electron systems in terms of one-electron quantities. ${ }^{41,42}$ Thus, the oneparticle density matrix $\Gamma\left(\mathbf{r}, \mathbf{r}^{\prime}\right)$ of a many-electron system can be easily obtained from the electron detachment Dyson orbitals

$$
\Gamma\left(\mathbf{r}, \mathbf{r}^{\prime}\right)=\sum_{k}\left|\gamma_{k}\right|^{2} \phi_{k}(\mathbf{r}) \phi_{k}^{*}\left(\mathbf{r}^{\prime}\right)
$$

and the total energy $E$ of the system can be obtained as

$$
E=\frac{1}{2} \sum_{k}\left|\gamma_{k}\right|^{2}\left(\left\langle\phi_{k}\left|\hat{h}_{1}\right| \phi_{k}\right\rangle+\varepsilon_{k}\right)
$$

which are reminiscent of the respective expressions in the mean-field theory. ${ }^{41,42}$ In the above equations, $\hat{h}_{1}$ is the oneelectron Hamiltonian, and $\varepsilon_{k}$ the $k$ th electron binding energy (the $k$ th ionization potential); the orbitals $\phi_{k}(\mathbf{r})$ are normalized as $\phi_{k}(\mathbf{r})=\varphi_{k}(\mathbf{r}) / \sqrt{\left|\gamma_{k}\right|^{2}}$.

Hence, as nicely demonstrated by Ortiz, ${ }^{41,42}$ the whole qualitative orbital theory can be formulated in terms of DOs. Conceptually, DOs are not the same as the commonly employed orbitals, e.g., MOs, although they may be very close to some forms of orbitals. ${ }^{38,50}$ One may say that DOs are not the one-electron functions that govern the electron motion inside the molecule and that they can not be interpreted as the orbitals from which an electron was ejected at the time of impingement of a photon in photoelectron spectroscopy or of an electron in electron momentum (e,2e) experiments. ${ }^{36}$ Nevertheless, it seems worth reiterating that their compatibility with the main concepts of quantum chemistry makes them convenient tools for analyzing the electronic structure; ${ }^{36,42}$ furthermore, they are experimentally verifiable. ${ }^{37,38,41,42}$

The Dyson orbitals and the respective electron binding energies (eBEs) can be obtained by solving the Dyson equation. $^{51,52}$ The simplest approximation to DOs and eBEs is given by the Hartree-Fock canonical orbitals and their energies. ${ }^{53} \mathrm{~A}$ more rigorous solution, which provides for certain account of electron correlation and orbital relaxation effects, is achieved in the electron propagator formalism. ${ }^{41,47-49}$ However, this methodology works well for weakly correlated initial states, whose electronic wave function is accurately approximated by a single electronic configuration (Slater determinant).

For strongly correlated (multiconfigurational) molecules, the Dyson orbitals are typically obtained from eq 1 directly, where the CASSCF $^{54}$ (CASSCF: complete active space SCF) wave functions from two separate computations-one for the initial and one for the final state- are used. ${ }^{55,56}$ The respective eBEs are calculated by taking the energy differences between the initial state and the final state; quite often the energies are obtained at a higher level of theory, e.g., CASPT2 ${ }^{57}$ (CASSCF augmented by the second-order many-body perturbation theory). Besides that such an approach lacks internal consistency, because the same level of treatment of the initial (neutral) and the final (ionized) states of the system is not likely to be guaranteed, obtaining higher ionization energies and the respective orbitals is becoming increasingly difficult, as it requires computation of highly excited states of the ionized system. On the bright side, the direct computation can be used in connection with any method that yields energy and the respective wave function.

Technically, the direct computation of eBEs and DOs can be used in connection with the response methods, such as the linear-response time-dependent DFT (LR-TDDFT) methodology $^{58-62}$ and mutations thereof. ${ }^{63-66}$ However, it was seldom used and predominantly used for obtaining the eBEs, not the DOs. Despite the advantages offered by the Dyson's orbital formalism, ${ }^{36,41,42}$ the response states of LR-TDDFT are typically analyzed in terms of transitions between the reference state orbitals or with the use of some purely mathematical constructs, such as NOs ${ }^{67,68}$ or NTOs. ${ }^{30}$

Herein, we propose a simple method of obtaining DOs and eBEs from a response formalism (e.g., TDDFT), which should enable construction of a physically motivated orbital picture for the ground and excited electronic states fully compatible with the main concepts of quantum chemistry. When doing so, we shall make use of the extended Koopmans' theorem (EKT) ${ }^{69-71}$ which enables obtaining the DOs and eBEs from the known (relaxed) density matrix and the molecular Lagrangian. $^{72}$

The main statement of EKT is that the Dyson orbitals and the electron binding energies span the eigensystem of the Lagrangian matrix transformed to the basis of the natural orbitals. $^{69-71}$ For electron detachment, EKT yields the exact first ionization potential ${ }^{73}$ and arbitrarily accurate higher ionization potentials. ${ }^{73,74}$ In the context of the Hartree-Fock method, EKT recovers the usual Koopmans' theorem, yielding the canonical HF orbitals and orbital energies as DOs and eBEs. However, EKT can easily incorporate multiconfigura- 
Table 1. Comparison of eBE (in eV) Obtained by EKT-MRSF with the (Negative) KS DFT Orbital Energies $\left(-\varepsilon\right.$, in eV) ${ }^{a}$

\begin{tabular}{|c|c|c|c|c|c|c|c|c|}
\hline \multirow[b]{2}{*}{ molecule } & \multirow[b]{2}{*}{ symm. $^{b}$} & \multirow[b]{2}{*}{ exptl..$^{17,83}$} & \multicolumn{3}{|c|}{ BH\&HLYP } & \multicolumn{3}{|c|}{ QTP17 } \\
\hline & & & $\mathrm{eBE}^{c}$ & $-\varepsilon^{d}$ & $\Delta E$ & eBE & $-\varepsilon$ & $\Delta E$ \\
\hline \multirow[t]{5}{*}{$\mathrm{H}_{2} \mathrm{O}$} & $1 b_{1}$ & 12.62 & 10.50 & 10.42 & 0.08 & 11.30 & 11.47 & -0.17 \\
\hline & $3 a_{1}$ & 14.74 & 12.43 & 12.45 & -0.02 & 13.42 & 13.47 & -0.05 \\
\hline & $1 b_{2}$ & 18.65 & 16.30 & 16.34 & -0.04 & 17.24 & 17.32 & -0.08 \\
\hline & $2 a_{1}$ & 32.61 & 31.02 & 30.98 & 0.04 & 32.46 & 32.52 & -0.06 \\
\hline & $1 a_{1}$ & 539.7 & 535.15 & 535.0 & 0.15 & 539.23 & 539.07 & 0.16 \\
\hline $\mathrm{RMSD}^{e}$ & & & 2.78 & 2.83 & 0.08 & 1.07 & 1.01 & 0.12 \\
\hline \multirow[t]{5}{*}{$\mathrm{H}_{2} \mathrm{CO}$} & $2 b_{2}$ & 10.90 & 9.02 & 9.32 & -0.30 & 9.89 & 10.12 & -0.23 \\
\hline & $1 b_{1}$ & 14.50 & 12.46 & 12.54 & -0.08 & 13.59 & 13.68 & -0.09 \\
\hline & $5 a_{1}$ & 16.10 & 14.64 & 14.60 & 0.04 & 15.89 & 15.80 & 0.09 \\
\hline & $1 b_{2}$ & 17.00 & 15.75 & 15.75 & 0.00 & 16.93 & 16.97 & -0.04 \\
\hline & $4 a_{1}$ & 21.40 & 19.99 & 19.89 & 0.10 & 21.03 & 20.98 & 0.05 \\
\hline RMSD & & & 1.64 & 1.58 & 0.15 & 0.64 & 0.92 & 0.12 \\
\hline \multirow[t]{5}{*}{$\mathrm{H}_{2} \mathrm{CS}$} & $3 b_{2}$ & 9.38 & 7.79 & 7.80 & -0.01 & 8.51 & 8.48 & 0.03 \\
\hline & $2 b_{1}$ & 11.76 & 9.94 & 10.02 & -0.08 & 11.62 & 10.83 & 0.79 \\
\hline & $7 a_{1}$ & 13.85 & 12.58 & 12.53 & 0.05 & 13.47 & 13.41 & 0.06 \\
\hline & $2 b_{2}$ & 15.20 & 14.78 & 14.83 & -0.05 & 15.65 & 15.76 & -0.11 \\
\hline & $6 a_{1}$ & 19.90 & 18.87 & 18.86 & 0.01 & 19.85 & 19.88 & -0.03 \\
\hline RMSD & & & 1.32 & 1.30 & 0.05 & 0.47 & 0.88 & 0.36 \\
\hline \multirow[t]{6}{*}{$\mathrm{C}_{2} \mathrm{H}_{4}$} & $1 b_{3 u}$ & 10.68 & 8.81 & 8.71 & 0.10 & 9.85 & 9.58 & 0.27 \\
\hline & $1 b_{3 g}$ & 12.80 & 11.49 & 11.48 & 0.01 & 12.10 & 12.12 & -0.02 \\
\hline & $3 a_{g}$ & 14.80 & 13.34 & 13.32 & 0.02 & 14.34 & 14.36 & -0.02 \\
\hline & $1 b_{2 u}$ & 16.00 & 14.80 & 14.79 & 0.01 & 15.79 & 15.81 & -0.02 \\
\hline & $2 \mathrm{~b}_{1 u}$ & 19.10 & 18.17 & 18.16 & 0.01 & 19.16 & 19.20 & -0.04 \\
\hline & $2 \mathrm{a}_{g}$ & 23.60 & 23.71 & 23.67 & 0.04 & 25.16 & 25.19 & -0.03 \\
\hline RMSD & & & 1.27 & 1.30 & 0.05 & 0.80 & 0.86 & 0.11 \\
\hline \multirow[t]{11}{*}{ furan } & $1 a_{2}$ & 9.00 & 7.41 & 7.45 & -0.04 & 8.26 & 8.18 & 0.08 \\
\hline & $2 b_{1}$ & 10.40 & 9.10 & 9.07 & 0.03 & 9.89 & 9.89 & 0.00 \\
\hline & $9 a_{1}$ & 13.00 & 12.14 & 12.14 & 0.00 & 13.04 & 13.05 & -0.01 \\
\hline & $8 a_{1}$ & 13.80 & 12.73 & 12.73 & 0.00 & 13.73 & 13.75 & -0.02 \\
\hline & $6 b_{2}$ & 14.40 & 13.18 & 13.17 & 0.01 & 14.03 & 14.02 & 0.01 \\
\hline & $5 b_{2}$ & 15.25 & 14.07 & 14.06 & 0.01 & 15.02 & 15.03 & -0.01 \\
\hline & $1 b_{1}$ & 15.60 & 14.32 & 14.32 & 0.00 & 15.78 & 15.78 & 0.00 \\
\hline & $7 a_{1}$ & 17.50 & 17.05 & 17.04 & 0.01 & 18.33 & 18.35 & -0.02 \\
\hline & $6 a_{1}$ & 18.80 & 17.94 & 17.92 & 0.02 & 18.92 & 18.92 & 0.00 \\
\hline & $4 b_{2}$ & 19.70 & 18.66 & 18.66 & 0.00 & 20.04 & 20.05 & -0.01 \\
\hline & $3 b_{2}$ & 23.00 & 23.18 & 23.15 & 0.03 & 24.75 & 24.76 & -0.01 \\
\hline RMSD & & & 1.07 & 1.08 & 0.02 & 0.67 & 0.68 & 0.03 \\
\hline \multirow[t]{10}{*}{ thiophene } & $1 a_{2}$ & 8.87 & 7.61 & 7.66 & -0.05 & 8.48 & 8.42 & 0.06 \\
\hline & $3 b_{1}$ & 9.52 & 8.08 & 8.06 & 0.02 & 8.70 & 8.75 & -0.05 \\
\hline & $11 a_{1}$ & 12.10 & 10.96 & 10.96 & 0.00 & 11.71 & 11.73 & -0.02 \\
\hline & $2 b_{1}$ & 12.70 & 12.00 & 11.99 & 0.01 & 13.11 & 13.14 & -0.03 \\
\hline & $7 b_{2}$ & 13.30 & 12.24 & 12.23 & 0.01 & 13.11 & 13.11 & 0.00 \\
\hline & $10 a_{1}$ & 13.90 & 12.52 & 12.51 & 0.01 & 13.46 & 13.46 & 0.00 \\
\hline & $6 b_{2}$ & 14.30 & 13.17 & 13.16 & 0.01 & 14.12 & 14.10 & 0.02 \\
\hline & $9 a_{1}$ & 16.60 & 16.11 & 16.11 & 0.00 & 17.31 & 17.30 & 0.01 \\
\hline & $5 b_{2}$ & 17.60 & 17.31 & 17.30 & 0.01 & 18.42 & 18.41 & 0.01 \\
\hline & $8 a_{1}$ & 18.30 & 17.67 & 17.65 & 0.02 & 18.78 & 18.77 & 0.01 \\
\hline RMSD & & & 1.02 & 1.03 & 0.02 & 0.53 & 0.52 & 0.03 \\
\hline \multirow[t]{11}{*}{ pyridine } & $1 a_{2}$ & 9.60 & 8.30 & 8.33 & -0.03 & 9.10 & 9.08 & 0.02 \\
\hline & $11 a_{1}$ & 9.75 & 8.91 & 8.91 & 0.00 & 9.63 & 9.65 & -0.02 \\
\hline & $2 b_{1}$ & 10.51 & 9.12 & 9.12 & 0.00 & 9.96 & 9.95 & 0.01 \\
\hline & $7 b_{2}$ & 12.61 & 11.78 & 11.78 & 0.00 & 12.63 & 12.64 & -0.01 \\
\hline & $1 b_{1}$ & 13.10 & 12.56 & 12.54 & 0.02 & 13.76 & 13.76 & 0.00 \\
\hline & $10 a_{1}$ & 13.80 & 13.04 & 13.05 & -0.01 & 13.98 & 14.00 & -0.02 \\
\hline & $6 b_{2}$ & 14.50 & 13.69 & 13.67 & 0.02 & 14.69 & 14.68 & 0.01 \\
\hline & $9 a_{1}$ & 15.90 & 14.97 & 14.96 & 0.01 & 15.84 & 15.83 & 0.01 \\
\hline & $5 b_{2}$ & 15.90 & 15.18 & 15.19 & -0.01 & 16.46 & 16.47 & -0.01 \\
\hline & $8 a_{1}$ & 17.40 & 16.63 & 16.62 & 0.01 & 17.90 & 17.90 & 0.00 \\
\hline & $4 b_{2}$ & 19.80 & 19.72 & 19.72 & 0.00 & 20.99 & 20.99 & 0.00 \\
\hline
\end{tabular}


Table 1. continued

\begin{tabular}{|c|c|c|c|c|c|c|c|c|}
\hline \multirow[b]{2}{*}{ molecule } & \multirow[b]{2}{*}{ symm. $^{b}$} & \multirow[b]{2}{*}{ exptl. ${ }^{17,83}$} & \multicolumn{3}{|c|}{ BH\&HLYP } & \multicolumn{3}{|c|}{ QTP17 } \\
\hline & & & $\mathrm{eBE}^{c}$ & $-\varepsilon^{d}$ & $\Delta E$ & $\mathrm{eBE}$ & $-\varepsilon$ & $\Delta E$ \\
\hline & $7 a_{1}$ & 20.60 & 19.73 & 19.72 & 0.01 & 21.06 & 21.07 & -0.01 \\
\hline & $3 b_{2}$ & 23.40 & 23.77 & 23.75 & 0.02 & 25.32 & 25.32 & 0.02 \\
\hline & $6 a_{1}$ & 24.50 & 25.07 & 25.08 & -0.01 & 26.68 & 26.68 & -0.01 \\
\hline & $5 a_{1}$ & 28.00 & 29.10 & 29.09 & 0.01 & 31.08 & 31.08 & 0.00 \\
\hline RMSD & & & 0.85 & 0.85 & 0.01 & 1.19 & 1.19 & 0.01 \\
\hline RMSD (all) & & & 1.35 & 1.36 & 0.06 & 0.86 & 0.91 & 0.12 \\
\hline
\end{tabular}

${ }^{a}$ The computations are performed for the $S_{0}$ states of benchmark molecules ${ }^{17,83}$ using the BH\&HLYP and QTP17 functionals. $\Delta E$ gives the difference between the KS DFT orbital energies and the EKT-MRSF eBEs. For comparison, the experimental ionization potentials are also given. ${ }^{b}$ Symmetry of the orbital undergoing ionization. ${ }^{c}$ eBE from EKT-MRSF. ${ }^{d}$ Negative of the KS DFT orbital energy, $-\varepsilon .{ }^{e}$ Root-mean-square deviation (RMSD) of the computed ionization energies from the experimental values; in the " $\Delta E$ " column, the RMSD of the MRSF Dyson orbital energies from the KS DFT orbital energies is given.

tional wave functions ${ }^{75}$ as well as the dynamic electron correlation. $^{72}$

At the practical level, EKT can be conveniently formulated as a generalized eigenproblem ${ }^{76}$

$$
\tilde{\mathbf{W}}^{K} \mathbf{C}^{K}=\mathbf{D}^{K} \mathbf{C}^{K} \boldsymbol{\varepsilon}^{K}
$$

where $\mathbf{D}^{K}$ and $\tilde{\mathbf{W}}^{K}$ are the relaxed density matrix ${ }^{77}$ (when the energy is not obtained variationally) and the Lagrangian matrix of the initial electronic state $K$ (e.g., neutral molecule in the state $K) .^{72,75}$ The $\mathbf{C}^{K}$ vectors collect the expansion coefficients of the Dyson orbitals $\varphi_{k}^{K}(\mathbf{r})$ in terms of the eigenvectors (natural orbitals) of the density matrix $\mathrm{D}^{K}$.

Hence, the DOs from EKT are superpositions of the molecular NOs. For relationship between DOs and other forms of orbitals, which can be obtained from the difference density matrices between the final and the initial states, see the recent articles by Ortiz and $\mathrm{Zalik}^{78}$ and Harb and Hratchian. ${ }^{79}$ The use of the EKT enables computation of all the eBEs and DOs of the system from a single calculation, rather than obtaining them one-by-one, as in the $\triangle$ SCF approach advocated in the latter articles.

The (relaxed) density matrix and the Lagrangian matrix are routinely obtained, when calculating the energy gradient $\partial E_{K} /$ $\partial \lambda$ with respect to the nuclear coordinates ${ }^{80}$

$$
\begin{aligned}
\frac{\partial E_{K}}{\partial \lambda}= & \operatorname{tr}\left(\mathbf{D}^{K} \frac{\partial \mathbf{h}_{1}}{\partial \lambda}\right)-\frac{1}{2} \operatorname{tr}\left(\tilde{\mathbf{W}}^{K} \frac{\partial \mathbf{S}}{\partial \lambda}\right) \\
& +\ldots \text { derivatives of the 2-electron terms }
\end{aligned}
$$

where $\partial \mathbf{h}_{1} / \partial \lambda$ and $\partial \mathbf{S} / \partial \lambda$ are the derivatives of the matrix of the one-electron integrals and of the overlap matrix, respectively. As the Lagrangian matrix imposes the orbital orthonormality constraints, the derivative of the constraints occurs in the energy gradient obtained with any method, where the orbital orthogonality is preserved. ${ }^{80}$

Hence, the DOs and the respective eBEs can be obtained with any computational method for which the analytical energy gradient is available. ${ }^{72,75}$ As an example, recently, EKT was implemented in connection with an ensemble density functional methodology, ${ }^{81,82}$ where a good agreement with the experiment was achieved for obtaining the ionization potentials and the electron affinities of a number of strongly correlated (i.e., multiconfigurational) systems. $^{83,84}$ It is, furthermore, surprising that EKT seems to have never been used in connection with response methodologies, such as LRTDDFT $^{59-62}$ and spin-flip (SF) TDDFT, ${ }^{63,64}$ where it could have provided means for a concise description of the (ground and) excited states in terms of the Dyson orbitals and the respective electron binding energies.

Here, the computation of DOs and eBEs by EKT is employed in connection with the mixed-reference SF-TDDFT (MRSF-TDDFT) methodology. ${ }^{65,66}$ MRSF-TDDFT (for brevity, MRSF) is a linear-response-type methodology ${ }^{59,60}$ which obtains the electronic states with singlet (as well as triplet) multiplicity as superpositions of the spin-flip orbital transitions (e.g., $\phi_{i}^{\alpha} \rightarrow \phi_{a}^{\beta}$ and $\phi_{j}^{\beta} \rightarrow \phi_{b}^{\alpha}$ ) originating from a triplet reference state. ${ }^{65}$ Because this allows including some important electronic configurations in the response space (e.g., the doubly excited configurations, which are missing in the traditional spin-conserving LR-TDDFT formalism ${ }^{59-62}$ ), MRSF is capable of including the strong multiconfigurational effects into the description of the ground and excited states $^{85-90}$ and accurately describing the conical intersections (real crossings) between the ground and excited singlet states. ${ }^{91}$ The great advantage of MRSF before the originally proposed SF-TDDFT formalism ${ }^{63,64}$ is that MRSF cures the notorious spin-contamination of the SF-TDDFT response states, $^{65}$ thus enabling clean discrimination between pure singlet and triplet states. For more detail of the MRSF method, see refs 65 and 66 and the Supporting Information.

By contrast to LR-TDDFT, all singlet states, the ground state included, are obtained in MRSF as response states. In the usual KS DFT/LR-TDDFT formalism, the ground electronic state (the reference state of LR-TDDFT) is obtained by variational optimization, i.e., by solving the KS SCF equations. Hence, one may wonder how (dis)similar are the orbitals provided by both formalisms.

In the following, the variationally obtained canonical KS orbitals from the usual KS DFT calculation will be compared with the Dyson orbitals from the $S_{0}$ response state of MRSF. To this end, the EKT-MRSF computation of DOs and eBEs was implemented in the local version of the GAMESS-US program $^{92,93}$ along the guidelines outlined in ref 83 (see the Supporting Information for more detail). For consistency, the KS DFT calculations were carried out using the same code. All the calculations presented in this work employed the 6$311 \mathrm{G}(\mathrm{d}, \mathrm{p})$ basis set. $^{94}$

The relaxed density matrix $\mathbf{D}^{K}$ and the Lagrangian $\tilde{\mathbf{W}}^{K}$ are routinely obtained during the calculation of the MRSF analytic energy gradient. ${ }^{66}$ The cost of the computation of these matrices is only a fraction of the cost of obtaining the vertical excitation energies by the response formalism. Hence, EKTMRSF is a very economic approach to obtaining all (not just a few) of the ionization energies of a molecule. 
It is also noteworthy that, as the relaxed density matrix $\mathbf{D}^{K}$ and the Lagrangian matrix $\tilde{\mathbf{W}}^{K}$ are obtained from solving a system of the coupled-perturbed equations and they incorporate the orbital response contributions, ${ }^{66}$ the resulting electronic states in MRSF are stationary. Indeed, these states satisfy the Hellmann-Feynman theorem ${ }^{95,96}$ in the sense that the expectation value of a one-electron operator (e.g., the dipole moment) evaluated with the relaxed density matrix is equal to the derivative of the total energy of the state with respect to the relevant external perturbation (e.g., an external electric field). Hence, the state is stationary with respect to the orbital rotations and the resulting Lagrangian is hermitian; which is required to rigorously invoke the EKT. ${ }^{97}$

A technical comment on solving the EKT eq 5 seems appropriate. The (relaxed) density matrix is near singular, as it has a (large) number of near zero eigenvalues. To bypass its singularity, in this work, the deflation method ${ }^{98}$ is invoked, where the Lagrangian $\tilde{\mathbf{W}}^{K}$ and density matrix $\mathbf{D}^{K}$ are projected onto the natural orbitals having the nonzero eigenvalues. ${ }^{72,83}$ As some NOs and their (near zero) eigenvalues are dropped, this may slightly disturb the normalization of the projected density matrix. As a consequence, some Dyson's orbitals may acquire norms slightly exceeding the unit norm, although the deviations are rather small and do not distort the shapes of the Dyson orbitals. This problem can be alleviated by using alternative approaches to solving generalized eigenvalue problems with a nearly singular metric, e.g., the shifting method. ${ }^{98}$ The utility of these approaches in connection with the EKT-MRSF method will be investigated in future work.

For a set of molecules used previously in refs 17 and 83, Table 1 presents the results of the KS DFT and the MRSF calculations obtained using two density functionals, BH\&HLYP ${ }^{99-101}$ and QTP17. ${ }^{102}$ The commonly employed approximate density functionals, because of the incorrect asymptotic behavior of the respective exchange-correlation potential, yield KS orbital energies uniformly shifted to more positive values than the true (negative) ionization potentials. $^{17,103}$ A series of recently developed QTP functionals attempts to cure this deficiency by requiring the (approximate) equality of the KS orbital energies and the ionization potentials. $^{102,104,105}$ Although the main focus in Table 1 is on comparing the orbital energies obtained from EKT with the KS DFT energies, the QTP17 functional is used here to underline that the agreement with the experiment can be improved in a systematic fashion. Indeed, the use of this functional improves the agreement with the experiment by $c a$. $0.5 \mathrm{eV}$ (see Table 1).

Perhaps, the most striking observation from Table 1 is that the eBEs of the MRSF DOs agree with the KS DFT MO energies within a few hundredths of an electronvolt, as illustrated by their differences $(\Delta E)$. The respective overall root-mean-square deviations (RMSDs) are 0.06 (BH\&HLYP) and $0.12 \mathrm{eV}$ (QTP17). This agreement seems surprising, as the $\mathrm{S}_{0}$ state in MRSF is obtained from a response computation, where it spans a number of electronic configurations built using the triplet reference state orbitals. This seems to be very different from the single-determinant KS DFT ground state. However, the orbital energies come out nearly the same.

The remarkable similarity between the EKT-MRSF and KS DFT orbital energies suggests that their respective orbital shapes, i.e., the shapes of the EKT-MRSF DOs and the KS DFT canonical orbitals, should be very similar as well. And they indeed are, as can be seen in Figure 1, where the valence

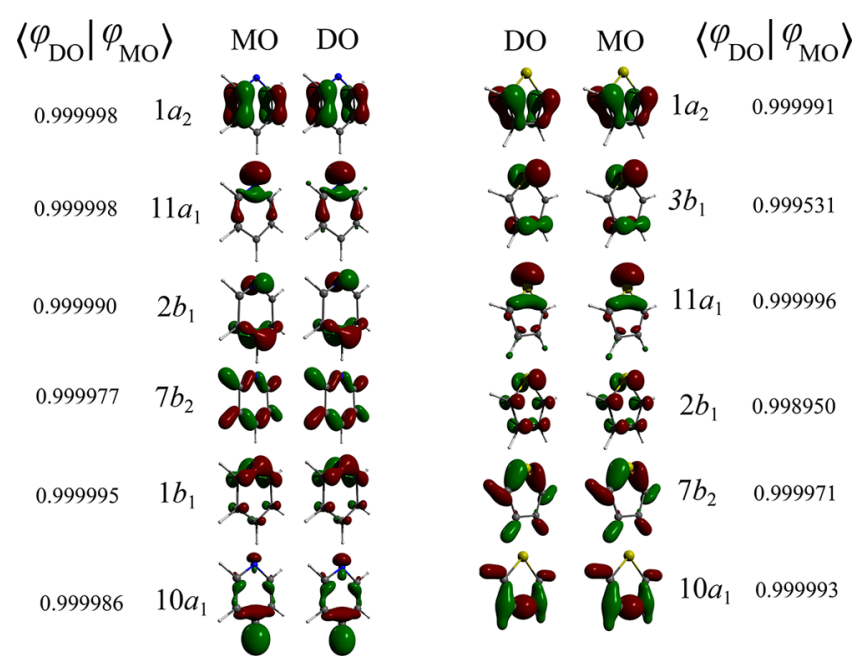

Figure 1. Comparison of EKT-MRSF DOs with KS-DFT MOs for pyridine (left) and thiophene (right) in their $S_{0}$ states. The boundary surfaces of the orbitals are plotted with the threshold of $10^{-1} \mathrm{bohr}^{-3}$. The values of the overlap integrals $\left\langle\phi_{\mathrm{DO}} \mid \varphi_{\mathrm{MO}}\right\rangle$ between the (normalized) DOs and the canonical MOs are also shown.

DOs and the KS DFT MOs of two molecules, pyridine and thiophene, are shown. The DOs $\phi^{\mathrm{DO}}(\mathbf{r})$ in Figure 1 were normalized, i.e., $\phi^{\mathrm{DO}}(\mathbf{r})=\varphi(\mathbf{r}) / \sqrt{|\gamma|^{2}}$, where $\varphi(\mathbf{r})$ and $|\gamma|^{2}$ are the raw Dyson orbitals and their norms as given in eqs 1 and 2, respectively. The overlap integrals $\left\langle\phi_{\mathrm{DO}} \mid \varphi_{\mathrm{MO}}\right\rangle$ between the (normalized) DOs and the canonical MOs deviate from unity only in the third digit!

The comparisons in Table 1 and Figure 1 confirm that the EKT-type computation in connection with a response methodology produces the same orbital picture of the ground electronic state as the traditional SCF (KS DFT) orbital optimization in terms of both orbital shapes and orbital energies. This suggests that a similar orbital picture could be obtained for the excited states as well, thus offering a simple way of analyzing the excited states according to commonly accepted chemical concepts, which was unavailable for the popular linear response methodologies, that is, in terms of orbitals that have not only shapes but energies as well. This should bridge the gap between the traditional chemical orbital concepts and the response calculations of the excited states.

Next, let us compare DOs of the ground and the excited electronic states. Figure 2 shows the DO diagram of thymine, which was recently studied by some of us, ${ }^{90}$ comparing the $S_{0}$, $S_{1}$, and $S_{2}$ states; see also Figure $S 2$ of the Supporting Information. The geometry of the $S_{0}$ state was optimized using the MRSF-BH\&HLYP/6-311G(d,p) method, and the vertically excited $S_{1}$ and $S_{2}$ states were calculated at the $S_{0}$ geometry. As the $S_{0}$ equilibrium geometry is planar, all the orbitals are classified by the irreducible representations of the $\mathrm{C}_{\mathrm{s}}$ group; see Figure S2 for the symmetry labels. The lowest ionization potentials (IPs) of the $S_{0}$ state obtained from the photoelectron spectra ${ }^{106,107}$ are shown in Figure S2 of the Supporting Information. When comparing the calculated $S_{0}$ eBEs with the IPs, one should bear in mind that the approximate density functionals, such as BH\&HLYP, yield the orbital energies uniformly shifted by a certain amount with respect to the exact IPs (see also Table 1). For thymine, this shift amounts to $c a .0 .8 \mathrm{eV}$, as seen from comparing the first 


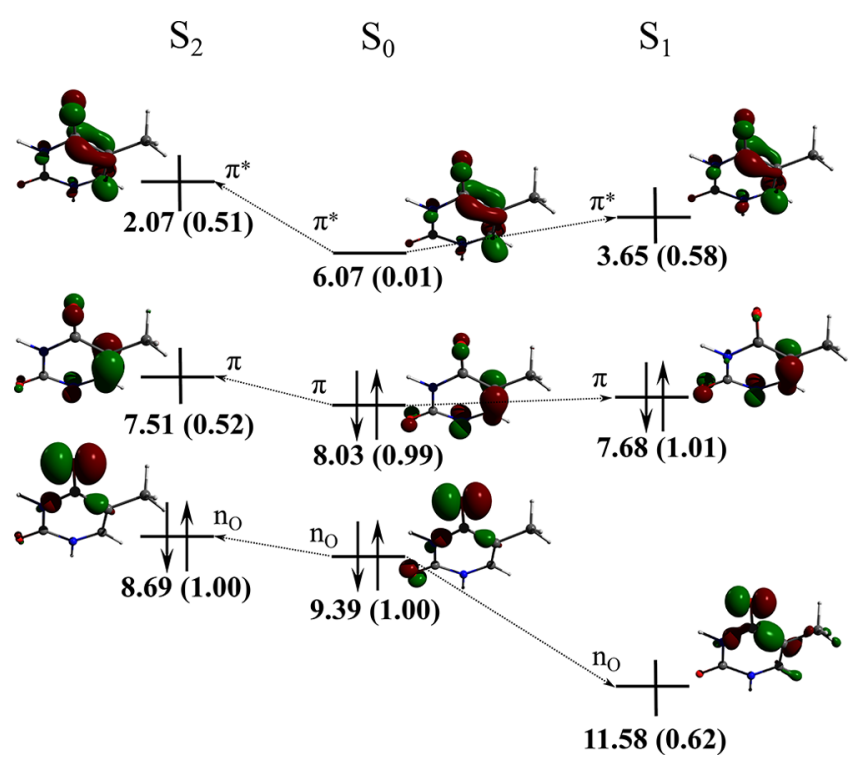

Figure 2. DO diagram of the $S_{0}, S_{1}$, and $S_{2}$ states of thymine calculated using MRSF-BH\&HLYP/6-311G(d,p) at the optimized $S_{0}$ equilibrium geometry. The eBEs of the DOs are given below the energy levels, and the respective norms $|\gamma|^{2}$ are given parenthetically. In the semioccupied orbitals, the arrowheads of the electron symbols were removed to underline that the depicted electronic configurations are not broken-symmetry single determinants but configuration state functions with the proper spin, $\left\langle S^{2}\right\rangle=0$. For the full diagram showing the other valence DOs of thymine, see Figure S2 of the Supporting Information.

experimental IP $(8.8 \mathrm{eV})$ with the first calculated eBE $(8.03$ $\mathrm{eV})$ (see Figure S2).

In the $S_{0}$ state, the uppermost DOs of thymine are the $\pi$ $\left(6 a^{\prime \prime}\right)$ and $\mathrm{n}_{\mathrm{O}}\left(27 a^{\prime}\right)$ orbitals, which are doubly occupied. The occupation of DOs follows from their norms $|\gamma|^{2}$, which are very near to one, for both uppermost DOs. The $\pi^{*}$ DO $\left(7 a^{\prime \prime}\right)$ is empty, as its norm $(0.01)$ is very small; hence, the probability of ionization for this orbital is negligible. This DO can be interpreted as corresponding to a shakeup ionization state, where the electron removal from the $6 a^{\prime \prime}$ orbital is accompanied by promotion of the remaining electron to the $7 a^{\prime \prime}$ orbital. However, its ionization energy occurs above the first ionization potential, which is counterintuitive for a shakeup satellite. This is a consequence of using an approximate density functional (BH\&HLYP) in the EKTMRSF calculations. With an alternative choice of the functional (QTP17), ${ }^{102}$ the shakeup satellite occurs $\mathrm{ca} .2 \mathrm{eV}$ below the first DO $\left(6 a^{\prime \prime}\right)$ (see Figure S3 in the Supporting Information). This points to certain problems with the currently available approximate density functionals. The study of this problem is however beyond the scope of the present work.

The shapes of the singly occupied DOs (the DOs with approximately half integer norms) in the $S_{1}$ and $S_{2}$ states are very close to the shapes of the respective NOs and NTOs obtained by the LR-TDDFT formalism (see Figure S4 of the Supporting Information). Hence, the DOs can be used in lieu of the NOs and NTOs.

The $S_{2}$ state is obtained by the $\pi \rightarrow \pi^{*}\left(6 a^{\prime \prime} \rightarrow 7 a^{\prime \prime}\right)$ orbital transition, and the respective DOs are singly occupied in this state; their norms are close to 0.5 , which is typical for single occupation. ${ }^{83}$ The electrons in the singly occupied DOs are bound weaker than in the $S_{0}$ state, and the energy difference between the first $S_{1}$ IP and the first $S_{0}$ IP $(5.96 \mathrm{eV})$ is approximately the same as the $\mathrm{S}_{0} \rightarrow \mathrm{S}_{2}$ vertical excitation energy (VEE, $5.98 \mathrm{eV}$ ) (see Figure S2). As ionization of both neutral states ends up in the ground state of the cation, the approximate equality of $\Delta \operatorname{IP}\left(S_{0}-S_{2}\right)=\operatorname{IP}\left(S_{0}\right)-\operatorname{IP}\left(S_{2}\right)$, and the VEE indicates relatively weak orbital relaxation.

The $S_{1}$ state of thymine is obtained by the $\mathrm{n}_{\mathrm{O}} \rightarrow \pi^{*}\left(27 a^{\prime} \rightarrow\right.$ $\left.7 a^{\prime \prime}\right)$ transition from the $S_{0}$ state. $\Delta \mathrm{IP}\left(S_{0}-S_{1}\right)(5.74 \mathrm{eV})$ deviates more from the $\operatorname{VEE}(5.91 \mathrm{eV})$ than in the $S_{2}$ state. This suggests that there is a more substantial orbital relaxation in the $\mathrm{n}_{\mathrm{O}} \pi^{*}$ state than in the $\pi \pi^{*}$ state. Intuitively, $\pi \pi^{*}$ might be expected to be the lowest excited state, as suggested by the orbital diagram of $S_{0}$. However, as the result of orbital relaxation, the $\mathrm{n}_{\mathrm{O}} \pi^{*}$ state falls below $\pi \pi^{*}$.

Indeed, the $\mathrm{n}_{\mathrm{O}} \rightarrow \pi^{*}$ transition causes depletion of the valence electron density on $\mathrm{O}$ in the ortho position to $\mathrm{CH}_{3}$. The total Mulliken charge on $\mathrm{O}$ varies from $-0.45 e$ in the $S_{0}$ state to $-0.28 \bar{e}$ in the $S_{1}$ state (the respective Löwdin charges are $-0.31 \bar{e}$ and $-0.04 \bar{e})$. As $\mathrm{O}$ becomes more positively charged, the respective lone pair orbital sinks below three other frontier orbitals and becomes $26 a^{\prime}$ (was $27 a^{\prime}$, in $S_{0}$ ) (see Figure S2 of the Supporting Information). The energy of the $\mathrm{n}_{\mathrm{O}} \mathrm{DO}$ is lowered by $c a .2 .2 \mathrm{eV}$, which provides a quantitative measure of the orbital relaxation, enabled by the use of Dyson's orbitals.

Besides the energetic criterion, the norms of the semioccupied DOs can be used to assess the degree of the orbital relaxation. As the orbital relaxation in the response states originates from mixing with the electronic configurations other than the single configuration corresponding to the main orbital transition, ${ }^{108}$ a stronger deviation of the norm from the halfinteger value $(0.5)$ indicates a stronger orbital relaxation. Hence, the $S_{1}$ state in Figure 2 undergoes stronger orbital relaxation with the norms of $0.58\left(\pi^{*}\right)$ and $0.62\left(n_{\mathrm{O}}\right)$, than the $\mathrm{S}_{2}$ state, where the Dyson norms are very close to the halfinteger values.

A comparison of the shapes of the DOs obtained in the ground and the excited states can reveal the detail of the orbital relaxation. Thus, the density differences $\Delta \mathrm{DO}_{k}=\left|\phi_{k}^{\mathrm{S}_{n}}\right|^{2}-\left|\phi_{k}^{S_{0}}\right|^{2}$ $\left(k=\pi, \pi^{*}, \mathrm{n}_{\mathrm{O}}\right)$ between the DOs in the $n$th excited state $(n=$ $1,2)$ and the ground state provide a visual measure ${ }^{109,110}$ and the overlap integral $\left\langle\phi_{k}^{\mathrm{S}_{n}} \mid \phi_{k}^{\mathrm{S}_{0}}\right\rangle$ quantifies the orbital shape variation shown in Figure 3. In the $S_{1}$ state, the most energetically relaxed $\mathrm{n}_{\mathrm{O}}$ DO $\left(26 a^{\prime}\right)$ exhibits the smallest overlap (0.808) and the largest visual difference with the $S_{0}$ state. Combined with the DO energies from the orbital

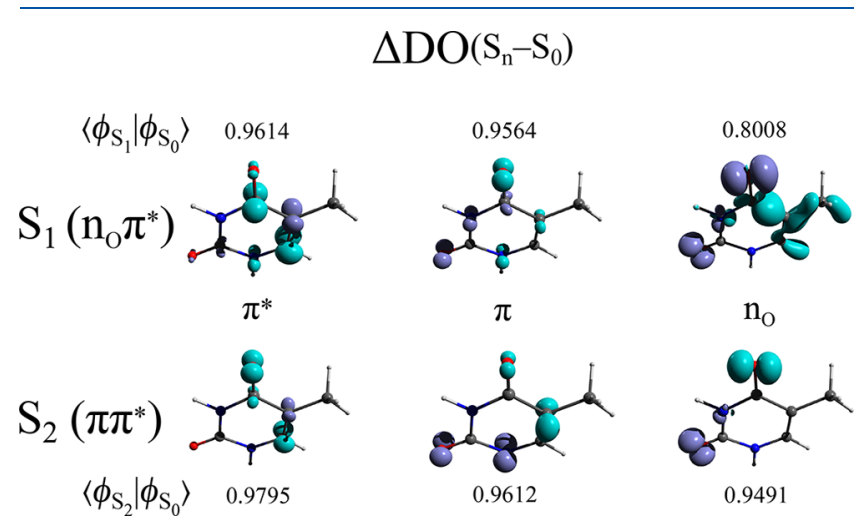

Figure 3. Comparison of the DO density differences $\Delta \mathrm{DO}$ mapped with the iso-value of $5 \times 10^{-3} \mathrm{bohr}^{-3}$. The overlap integrals $\left\langle\phi_{k}^{S_{n}} \mid \phi_{k}^{S_{0}}\right\rangle$ between the ground and excited state DOs are also shown. 
diagram in Figure 2 (and in Figure S2), this analysis furnishes the full orbital picture of the electronic excitations.

In conclusion, herein we presented a number of arguments in favor of using the Dyson orbitals for building a consistent orbital picture of the excited and response states obtained by response methodologies. Indeed, as argued in a number of essays and review articles, ${ }^{36,41,42}$ DOs have a number of attractive features - they have not only shapes, but the energies as well-which enable generalization of chemical concepts formulated for simple SCF methodologies to correlated and response methods. Employing the fact that the $S_{0}$ state in MRSF-TDDFT is obtained as a response state allows us to exhibit that the ground-state DOs and eBEs agree remarkably well with the MOs and their energies obtained in a usual KS DFT calculation. For the excited states, the shapes of the DOs closely match the shapes of NTOs, ${ }^{30}$ which are often used for analysis of the orbital transitions. However, the DOs of the excited states have energies, which enable building orbital correlation diagrams providing a lucid interpretation of the nature of the electronic transitions in the excited states. Combined with the ease of obtaining the DOs and eBEs through the use of the EKT, which requires only the knowledge of some ingredients (density matrix and Lagrangian) already available in the analytical energy gradient, this lays out a basis for developing a general approach to describing the electronic structure of the ground and excited states using traditional chemical concepts. In particular, Dyson's orbitals from popular linear response methodologies, such as LRTDDFT, SF-TDDFT, and MRSF-TDDFT, widely used in computational practice, will enable communication of the complicated analysis of the electronic structure of the excited and multiconfigurational electronic states in the language easily understood by the wide community of chemists.

\section{ASSOCIATED CONTENT}

\section{SI Supporting Information}

The Supporting Information is available free of charge at https://pubs.acs.org/doi/10.1021/acs.jpclett.1c02494.

Description of the MRSF-TDDFT methodology and the EKT-MRSF formalism, full orbital diagram of the ground and excited states of thymine, and comparison of DOs with NOs and NTOs (PDF)

\section{AUTHOR INFORMATION}

\section{Corresponding Authors}

Sason Shaik - The Lise Meitner-Minerva Center for Computational Quantum Chemistry, Institute of Chemistry, The Hebrew University of Jerusalem, Jerusalem 91904, Israel; Email: sason.shaik@gmail.com

Michael Filatov - Department of Chemistry, Kyungpook National University, Daegu 702-701, South Korea; ㅇo orcid.org/0000-0002-1541-739X; Email: mike.filatov@ gmail.com

Cheol Ho Choi - Department of Chemistry, Kyungpook National University, Daegu 702-701, South Korea; 다이.org/0000-0002-8757-1396; Email: cchoi@ knu.ac.kr

\section{Authors}

Vladimir Pomogaev - Department of Chemistry, Kyungpook National University, Daegu 702-701, South Korea; (1) orcid.org/0000-0003-4774-3998
Seunghoon Lee - Division of Chemistry and Chemical Engineering, California Institute of Technology, Pasadena, California 91125, United States; orcid.org/0000-00033665-587X

Complete contact information is available at:

https://pubs.acs.org/10.1021/acs.jpclett.1c02494

\section{Notes}

The authors declare no competing financial interest.

\section{ACKNOWLEDGMENTS}

This work was supported by the Samsung Science and Technology Foundations Grant SSTF-BA1701-12 (to C.H.C.) for fundamental theory developments and the NRF Grants 2019H1D3A2A02102948 (to M.F.) and 2020R1A2C2008246 and 2020R1A5A1019141 (to C.H.C.) funded by the Ministry of Science and ICT for applications of the developed methodologies.

\section{REFERENCES}

(1) Lennard-Jones, J. E. The electronic structure of some diatomic molecules. Trans. Faraday Soc. 1929, 25, 668-686.

(2) Hückel, E. Quantentheoretische Beiträge zum Benzolproblem. I. Die Elektronenkonfiguration des Benzols und verwandter Verbindungen. Eur. Phys. J. A 1931, 70, 204-286.

(3) Hückel, E. Theory of free radicals of organic chemistry. Trans. Faraday Soc. 1934, 30, 40-52.

(4) Coulson, C. A. Self-consistent field for molecular hydrogen. Math. Proc. Cambridge Philos. Soc. 1938, 34, 204-212.

(5) Hoffmann, R. Building Bridges Between Inorganic and Organic Chemistry (Nobel Lecture). Angew. Chem., Int. Ed. Engl. 1982, 21, $711-724$.

(6) Dewar, M. J. S. A Molecular Orbital Theory of Organic Chemistry. I. General Principles. J. Am. Chem. Soc. 1952, 74, 33413345.

(7) Roothaan, C. C. J. New Developments in Molecular Orbital Theory. Rev. Mod. Phys. 1951, 23, 69-89.

(8) Hall, G. G.; Lennard-Jones, J. E. The molecular orbital theory of chemical valency VIII. A method of calculating ionization potentials. Proc. R. Soc. Ser. A. Math. Phys. Sci. 1951, 205, 541-552.

(9) Pople, J. A. Molecular orbital methods in organic chemistry. Acc. Chem. Res. 1970, 3, 217-223.

(10) Hehre, W. J. Ab initio molecular orbital theory. Acc. Chem. Res. 1976, 9, 399-406.

(11) Löwdin, P.-O. Quantum Theory of Many-Particle Systems. II. Study of the Ordinary Hartree-Fock Approximation. Phys. Rev. 1955, 97, 1490-1508.

(12) Koopmans, T. Über die Zuordnung von Wellenfunktionen und Eigenwerten zu den Einzelnen Elektronen Eines Atoms. Physica 1934, $1,104-113$.

(13) Frost, A. A.; Braunstein, J.; Schwemer, W. Correlated Molecular Orbitals. J. Am. Chem. Soc. 1948, 70, 3292-3295.

(14) Shavitt, I. In Modern Theoretical Chemistry Vol. 3: Methods of Electronic Structure Theory; Schaefer, H. F., III, Ed.; Plenum: New York, 1977; pp 189-275.

(15) Hohenberg, P.; Kohn, W. Inhomogeneous Electron Gas. Phys. Rev. 1964, 136, B864-B871.

(16) Kohn, W.; Sham, L. J. Self-Consistent Equations Including Exchange and Correlation Effects. Phys. Rev. 1965, 140, A1133A1138.

(17) Chong, D. P.; Gritsenko, O. V.; Baerends, E. J. Interpretation of the Kohn-Sham orbital energies as approximate vertical ionization potentials. J. Chem. Phys. 2002, 116, 1760-1772.

(18) Giesbertz, K. J. H.; Baerends, E.-J. Aufbau derived from a unified treatment of occupation numbers in Hartree-Fock, KohnSham, and natural orbital theories with the Karush-Kuhn-Tucker 
conditions for the inequality constraints $\mathrm{ni} \leq 1$ and $\mathrm{ni} \geq 0$. J. Chem. Phys. 2010, 132, 194108.

(19) Löwdin, P.-O. Quantum Theory of Many-Particle Systems. I. Physical Interpretations by Means of Density Matrices, Natural SpinOrbitals, and Convergence Problems in the Method of Configurational Interaction. Phys. Rev. 1955, 97, 1474-1489.

(20) Löwdin, P.-O.; Shull, H. Natural Orbitals in the Quantum Theory of Two-Electron Systems. Phys. Rev. 1956, 101, 1730-1739.

(21) Löwdin, P.-O. Expansion Theorems for the Total Wave Function and Extended Hartree-Fock Schemes. Rev. Mod. Phys. 1960, $32,328-334$.

(22) Kobe, D. H. Natural Orbitals, Divergences, and Variational Principles. J. Chem. Phys. 1969, 50, 5183-5194.

(23) Suaud, N.; Malrieu, J.-P. Natural molecular orbitals: limits of a Löwdin's conjecture. Mol. Phys. 2017, 115, 2684-2695.

(24) Bohr, N. Atomic Structure. Nature 1921, 107, 104-107.

(25) Pauli, W. Über den Zusammenhang des Abschlusses der Elektronengruppen im Atom mit der Komplexstruktur der Spektren. Z. Phys. 1925, 31, 765-783.

(26) Woodward, R. B.; Hoffmann, R. Stereochemistry of Electrocyclic Reactions. J. Am. Chem. Soc. 1965, 87, 395-397.

(27) Woodward, R. B.; Hoffmann, R. The Conservation of Orbital Symmetry. Angew. Chem., Int. Ed. Engl. 1969, 8, 781-853.

(28) Fukui, K. The Role of Frontier Orbitals in Chemical Reactions (Nobel Lecture). Angew. Chem., Int. Ed. Engl. 1982, 21, 801-809.

(29) Krylov, A. I. From orbitals to observables and back. J. Chem. Phys. 2020, 153, 080901.

(30) Martin, R. L. Natural transition orbitals. J. Chem. Phys. 2003, 118, 4775-4777.

(31) Etienne, T. Transition matrices and orbitals from reduced density matrix theory. J. Chem. Phys. 2015, 142, 244103.

(32) Scerri, E. R. Have Orbitals Really Been Observed? J. Chem. Educ. 2000, 77, 1492.

(33) Wang, S.-G.; Schwarz, W. H. E. On Closed-Shell Interactions, Polar Covalences, d Shell Holes, and Direct Images of Orbitals: The Case of Cuprite. Angew. Chem., Int. Ed. 2000, 39, 1757-1761.

(34) Schwarz, W. H. E. Measuring Orbitals: Provocation or Reality? Angew. Chem., Int. Ed. 2006, 45, 1508-1517.

(35) Ogilvie, J. Is a molecular orbital measurable by means of tomographic imaging? Found. Chem. 2011, 13, 87-91.

(36) Truhlar, D. G.; Hiberty, P. C.; Shaik, S.; Gordon, M. S.; Danovich, D. Orbitals and the Interpretation of Photoelectron Spectroscopy and (e,2e) Ionization Experiments. Angew. Chem., Int. Ed. 2019, 58, 12332-12338.

(37) Brion, C.; Cooper, G.; Zheng, Y.; Litvinyuk, I.; McCarthy, I. Imaging of orbital electron densities by electron momentum spectroscopy - a chemical interpretation of the binary $(\mathrm{e}, 2 \mathrm{e})$ reaction. Chem. Phys. 2001, 270, 13-30.

(38) Brion, C.; Wolfe, S.; Shi, Z.; Cooper, G.; Zheng, Y. J. An investigation of hybridization and the orbital models of molecular electronic structure for $\mathrm{CH} 4, \mathrm{NH} 3$, and H2O. Can. J. Chem. 2017, 95, 1314-1322.

(39) Berglund, C. N.; Spicer, W. E. Photoemission Studies of Copper and Silver: Theory. Phys. Rev. 1964, 136, A1030-A1044.

(40) Schlitt, D. W.; Stutz, C. An Instructive Example of the Sudden Approximation in Quantum Mechanics. Am. J. Phys. 1970, 38, 70-75.

(41) Ortiz, J. V. Brueckner orbitals, Dyson orbitals, and correlation potentials. Int. J. Quantum Chem. 2004, 100, 1131-1135.

(42) Ortiz, J. V. Dyson-orbital concepts for description of electrons in molecules. J. Chem. Phys. 2020, 153, 070902.

(43) Goscinski, O.; Lindner, P. Natural Spin-Orbitals and Generalized Overlap Amplitudes. J. Math. Phys. 1970, 11, 1313-1317.

(44) Pickup, B.; Goscinski, O. Direct calculation of ionization energies. Mol. Phys. 1973, 26, 1013-1035.

(45) Pickup, B. T. On the theory of fast photoionization processes. Chem. Phys. 1977, 19, 193-208.

(46) Öhrn, Y.; Born, G. In Molecular Electron Propagator Theory and Calculations; Löwdin, P.-O., Ed.; Advances in Quantum Chemistry; Academic Press, 1981; Vol. 13; pp 1-88.
(47) Linderberg, J.; Öhrn, Y. Propagators in Quantum Chemistry; Academic Press: New York, 1973.

(48) Purvis, G. D.; Öhrn, Y. Accurate molecular ionization potentials from the electron propagator. Int. J. Quantum Chem. 1977, 12, 359363.

(49) Ortiz, J. V. Electron propagator theory: an approach to prediction and interpretation in quantum chemistry. WIREs Comp. Mol. Sci. 2013, 3, 123-142.

(50) Duffy, P.; Chong, D. P.; Casida, M. E.; Salahub, D. R. Assessment of Kohn-Sham density-functional orbitals as approximate Dyson orbitals for the calculation of electron-momentum-spectroscopy scattering cross sections. Phys. Rev. A: At., Mol., Opt. Phys. 1994, 50, 4707-4728.

(51) Dyson, F. J. The $S$ Matrix in Quantum Electrodynamics. Phys. Rev. 1949, 75, 1736-1755.

(52) Schwinger, J. On the Green's functions of quantized fields. I. Proc. Natl. Acad. Sci. U. S. A. 1951, 37, 452-455.

(53) Díaz-Tinoco, M.; Corzo, H. H.; Pawłowski, F.; Ortiz, J. V. Do Dyson Orbitals resemble canonical Hartree-Fock orbitals? Mol. Phys. 2019, 117, 2275-2283.

(54) Roos, B. O. In Ab Initio Methods in Quantum Chemistry II; Lawley, K. P., Ed.; John Wiley and Sons: New York, 1987; pp 399446.

(55) Melania Oana, C.; Krylov, A. I. Dyson orbitals for ionization from the ground and electronically excited states within equation-ofmotion coupled-cluster formalism: Theory, implementation, and examples. J. Chem. Phys. 2007, 127, 234106.

(56) Thompson, A. L.; Martínez, T. J. Time-resolved photoelectron spectroscopy from first principles: Excited state dynamics of benzene. Faraday Discuss. 2011, 150, 293-311.

(57) Andersson, K.; Malmqvist, P.; Roos, B. O. Second order perturbation theory with a complete active space self consistent field reference function. J. Chem. Phys. 1992, 96, 1218-1226.

(58) Runge, E.; Gross, E. K. U. Density-Functional Theory for Time-Dependent Systems. Phys. Rev. Lett. 1984, 52, 997-1000.

(59) Casida, M. E. In Recent Advances in Density Functional Methods; Chong, D. P., Ed.; Recent Advances in Computational Chemistry; World Scientific: Singapore, 1995; Vol. 1; pp 155-192.

(60) Casida, M. E.; Jamorski, C.; Bohr, F.; Guan, J. G.; Salahub, D. R. Optical properties from density-functional theory. ACS Symp. Ser. 1996, 628, 145-163. Symposium on Nonlinear Optical Materials Theory and Modeling, at the 208th National Meeting of the American-Chemical-Society, Washington, DC, Aug 21-25, 1994.

(61) Burke, K.; Werschnik, J.; Gross, E. K. U. Time-dependent density functional theory: Past, present, and future. J. Chem. Phys. 2005, 123, 062206.

(62) Casida, M.; Huix-Rotllant, M. Progress in Time-Dependent Density-Functional Theory. Annu. Rev. Phys. Chem. 2012, 63, 287323. PMID: 22242728.

(63) Shao, Y.; Head-Gordon, M.; Krylov, A. I. The spin-flip approach within time-dependent density functional theory: Theory and applications to diradicals. J. Chem. Phys. 2003, 118, 4807-4818.

(64) Wang, F.; Ziegler, T. Time-dependent density functional theory based on a noncollinear formulation of the exchange-correlation potential. J. Chem. Phys. 2004, 121, 12191-12196.

(65) Lee, S.; Filatov, M.; Lee, S.; Choi, C. H. Eliminating spincontamination of spin-flip time dependent density functional theory within linear response formalism by the use of zeroth-order mixedreference (MR) reduced density matrix. J. Chem. Phys. 2018, 149, 104101.

(66) Lee, S.; Kim, E. E.; Lee, S.; Choi, C. H. Efficient implementations of analytic energy gradient for mixed-reference spin-flip time-dependent density functional theory (MRSF-TDDFT). J. Chem. Phys. 2019, 150, 184111.

(67) Surján, P. R. Natural orbitals in CIS and singular-value decomposition. Chem. Phys. Lett. 2007, 439, 393-394.

(68) Luzanov, A. V.; Zhikol, O. A. Electron invariants and excited state structural analysis for electronic transitions within CIS, RPA, and TDDFT models. Int. J. Quantum Chem. 2009, 110, 902-924. 
(69) Morrell, M. M.; Parr, R. G.; Levy, M. Calculation of ionization potentials from density matrices and natural functions, and the longrange behavior of natural orbitals and electron density. J. Chem. Phys. $1975,62,549-554$.

(70) Day, O. W.; Smith, D. W.; Garrod, C. A generalization of the hartree-fock one-particle potential. Int. J. Quantum Chem. 1974, 8, 501-509.

(71) Smith, D. W.; Day, O. W. Extension of Koopmans' theorem. I. Derivation. J. Chem. Phys. 1975, 62, 113-114.

(72) Cioslowski, J.; Piskorz, P.; Liu, G. Ionization potentials and electron affinities from the extended Koopmans' theorem applied to energy-derivative density matrices: The EKTMPn and EKTQCISD methods. J. Chem. Phys. 1997, 107, 6804-6811.

(73) Vanfleteren, D.; Van Neck, D.; Ayers, P. W.; Morrison, R. C.; Bultinck, P. Exact ionization potentials from wavefunction asymptotics: The extended Koopmans' theorem, revisited. J. Chem. Phys. 2009, 130, 194104.

(74) Ernzerhof, M. Validity of the Extended Koopmans' Theorem. J. Chem. Theory Comput. 2009, 5, 793-797.

(75) Morrison, R. C.; Liu, G. Extended Koopmans' theorem: Approximate ionization energies from MCSCF wave functions. J. Comput. Chem. 1992, 13, 1004-1010.

(76) Peters, G.; Wilkinson, J. H. Ax $=\lambda \mathrm{Bx}$ and the Generalized Eigenproblem. SIAM J. Num. Analysis 1970, 7, 479-492.

(77) Perera, S. A.; Watts, J. D.; Bartlett, R. J. A theoretical study of hyperfine coupling constants. J. Chem. Phys. 1994, 100, 1425-1434.

(78) Ortiz, J. V.; Zalik, R. A. Eigenvalues of uncorrelated, densitydifference matrices and the interpretation of $\Delta$-self-consistent-field calculations. J. Chem. Phys. 2020, 153, 114122.

(79) Harb, H.; Hratchian, H. P. $\triangle$ SCF Dyson orbitals and pole strengths from natural ionization orbitals. J. Chem. Phys. 2021, 154, 084104.

(80) Pulay, P. Ab initio calculation of force constants and equilibrium geometries in polyatomic molecules. Mol. Phys. 1969, 17, 197-204.

(81) Filatov, M. Spin-restricted ensemble-referenced Kohn-Sham method: basic principles and application to strongly correlated ground and excited states of molecules. WIREs Comput. Mol. Sci. 2015, 5, $146-167$.

(82) Filatov, M. In Density-functional methods for excited states; Ferré, N., Filatov, M., Huix-Rotllant, M., Eds.; Top. Curr. Chem.; Springer: Heidelberg, 2016; Vol. 368; pp 97-124.

(83) Filatov, M.; Lee, S.; Choi, C. H. Computation of Molecular Ionization Energies Using an Ensemble Density Functional Theory Method. J. Chem. Theory Comput. 2020, 16, 4489-4504.

(84) Filatov, M.; Lee, S.; Nakata, H.; Choi, C. H. Computation of Molecular Electron Affinities Using an Ensemble Density Functional Theory Method. J. Phys. Chem. A 2020, 124, 7795-7804.

(85) Horbatenko, Y.; Lee, S.; Filatov, M.; Choi, C. H. Performance Analysis and Optimization of Mixed-Reference Spin-Flip TimeDependent Density Functional Theory (MRSF-TDDFT) for Vertical Excitation Energies and Singlet-Triplet Energy Gaps. J. Phys. Chem. A 2019, 123, 7991-8000.

(86) Horbatenko, Y.; Lee, S.; Filatov, M.; Choi, C. H. How Beneficial Is the Explicit Account of Doubly-Excited Configurations in Linear Response Theory? J. Chem. Theory Comput. 2021, 17, 975984.

(87) Horbatenko, Y.; Sadiq, S.; Lee, S.; Filatov, M.; Choi, C. H. Mixed-Reference Spin-Flip Time-Dependent Density Functional Theory (MRSF-TDDFT) as a Simple yet Accurate Method for Diradicals and Diradicaloids. J. Chem. Theory Comput. 2021, 17, 848859.

(88) Baek, Y. S.; Lee, S.; Filatov, M.; Choi, C. H. Optimization of Three State Conical Intersections by Adaptive Penalty Function Algorithm in Connection with the Mixed-Reference Spin-Flip TimeDependent Density Functional Theory Method (MRSF-TDDFT). J. Phys. Chem. A 2021, 125, 1994-2006.

(89) Lee, S.; Horbatenko, Y.; Filatov, M.; Choi, C. H. Fast and Accurate Computation of Nonadiabatic Coupling Matrix Elements
Using the Truncated Leibniz Formula and Mixed-Reference Spin-Flip Time-Dependent Density Functional Theory. J. Phys. Chem. Lett. 2021, 12, 4722-4728.

(90) Park, W.; Lee, S.; Huix-Rotllant, M.; Filatov, M.; Choi, C. H. Impact of the Dynamic Electron Correlation on the Unusually Long Excited-State Lifetime of Thymine. J. Phys. Chem. Lett. 2021, 12, 4339-4346.

(91) Lee, S.; Shostak, S.; Filatov, M.; Choi, C. H. Conical Intersections in Organic Molecules: Benchmarking Mixed-Reference Spin-Flip Time-Dependent DFT (MRSF-TD-DFT) vs Spin-Flip TDDFT. J. Phys. Chem. A 2019, 123, 6455-6462.

(92) Schmidt, M. W.; Baldridge, K. K.; Boatz, J. A.; Elbert, S. T.; Gordon, M. S.; Jensen, J. J.; Koseki, S.; Matsunaga, N.; Nguyen, K. A.; $\mathrm{Su}, \mathrm{S}$.; et al. J. Comput. Chem. 1993, 14, 1347-1363.

(93) Gordon, M.; Schmidt, M. In Theory and Applications of Computational Chemistry, the First Forty Years; Dykstra, C. E., Frenking, G., Kim, K. S., Scuseria, G. E., Eds.; Elsevier: Amsterdam, 2005; pp 1167-1189.

(94) Krishnan, R.; Binkley, J. S.; Seeger, R.; Pople, J. A. Selfconsistent molecular orbital methods. XX. A basis set for correlated wave functions. J. Chem. Phys. 1980, 72, 650-654.

(95) Hellmann, H. Einführung in die Quantenchemie; Franz Deuticke: Leipzig, 1937; p 285.

(96) Feynman, R. P. Forces in Molecules. Phys. Rev. 1939, 56, 340343.

(97) Davidson, E. R.; Ortiz, J. V.; Staroverov, V. N. Complete-activespace extended Koopmans theorem method. J. Chem. Phys. 2021, 155, 051102 .

(98) Hemmatiyan, S.; Sajjan, M.; Schlimgen, A. W.; Mazziotti, D. A. Excited-State Spectra of Strongly Correlated Molecules from a Reduced-Density-Matrix Approach. J. Phys. Chem. Lett. 2018, 9, 5373-5378.

(99) Becke, A. D. A New Mixing of Hartree-Fock and Local DensityFunctional Theories. J. Chem. Phys. 1993, 98, 1372-1377.

(100) Becke, A. D. Density-Functional Exchange-Energy Approximation with Correct Asymptotic Behavior. Phys. Rev. A: At., Mol., Opt. Phys. 1988, 38, 3098-3100.

(101) Lee, C.; Yang, W.; Parr, R. G. Development of the ColleSalvetti Correlation-Energy Formula into a Functional of the Electron Density. Phys. Rev. B: Condens. Matter Mater. Phys. 1988, 37, 785789.

(102) Jin, Y.; Bartlett, R. J. Accurate computation of X-ray absorption spectra with ionization potential optimized global hybrid functional. J. Chem. Phys. 2018, 149, 064111.

(103) Politzer, P.; Abu-Awwad, F. A comparative analysis of Hartree-Fock and Kohn-Sham orbital energies. Theor. Chem. Acc. 1998, 99, 83-87.

(104) Verma, P.; Bartlett, R. J. Increasing the applicability of density functional theory. IV. Consequences of ionization-potential improved exchange-correlation potentials. J. Chem. Phys. 2014, 140, 18A534.

(105) Jin, Y.; Bartlett, R. J. The QTP family of consistent functionals and potentials in Kohn-Sham density functional theory. J. Chem. Phys. 2016, 145, 034107.

(106) Lin, J.; Yu, C.; Peng, S.; Akiyama, I.; Li, K.; Lee, L. K.; LeBreton, P. R. Ultraviolet photoelectron studies of the ground-state electronic structure and gas-phase tautomerism of purine and adenine. J. Am. Chem. Soc. 1980, 102, 4627-4631.

(107) Fulfer, K. D.; Hardy, D.; Aguilar, A. A.; Poliakoff, E. D. Highresolution photoelectron spectra of the pyrimidine-type nucleobases. J. Chem. Phys. 2015, 142, 224310.

(108) Kimber, P.; Plasser, F. Toward an understanding of electronic excitation energies beyond the molecular orbital picture. Phys. Chem. Chem. Phys. 2020, 22, 6058-6080.

(109) Mayer, I. Using singular value decomposition for a compact presentation and improved interpretation of the CIS wave function. Chem. Phys. Lett. 2007, 437, 284-286.

(110) Pomogaev, V. A.; Ramazanov, R. R.; Ruud, K.; Artyukhov, V. Y. Insight into the fluorescence quenching of Trp214 at HSA by the 
Dimetridazole ligand from simulation. J. Photochem. Photobiol., A 2018, 354, 86-100. 